\title{
Morphological studies of the cytotoxicity of Trichomonas vaginalis to normal human vaginal epithelial cells in vitro
}

\author{
S E RASMUSSEN,* M H NIELSEN, $\uparrow$ I LIND, $\ddagger$ AND J M RHODES* \\ From the *International Escherichia and Klebsiella Centre, Statens Seruminstitut, the $\dagger$ Institute of \\ Pathological Anatomy, Copenhagen University, and the $\ddagger$ Neisseria Department, Statens Seruminstitut, \\ Copenhagen, Denmark
}

SUMMARY In vitro cultured monolayers of normal human vaginal epithelial cells were incubated with axenic cultures of Trichomonas vaginalis. Two strains freshly isolated from patients with trichomoniasis and one strain that had been maintained in axenic culture for several years were studied.

Freshly isolated trichomonads showed amoeboid movements, adherence to epithelial cell surfaces, and were cytotoxic to epithelial cells in vitro. In contrast, the laboratory strain maintained for years in axenic culture did not adhere to the epithelial cell monolayer and was only cytotoxic at a concentration 100 times that of freshly isolated trichomonads.

Electron microscopy of monolayers exposed to $T$ vaginalis for 24 hours showed that all epithelial cells in intimate contact with trichomonads had more or less disintegrated, whereas in monolayers exposed for six hours some of the epithelial cells in contact with $T$ vaginalis were normal.

$T$ vaginalis organisms with amoeboid morphology contained a dense network of microfilaments in the part of the trichomonad that was in contact with an epithelial cell. Occasionally a pseudopodium was projected into the cytoplasm of disintegrated epithelial cells.

\section{Introduction}

Trichomoniasis, a disease of the urogenital tract caused by Trichomonas vaginalis, affects both men and women and is the most prevalent protozoan infection in man. The symptoms of trichomoniasis may vary, either because different strains of $T$ vaginalis appear to have different pathogenic capabilities or because of variation in the host response. ${ }^{1}$ For example, though trichomoniasis in women is usually characterised by a copious, evil smelling discharge, about $25 \%$ of women harbouring trichomonads have

Address for reprints: Dr J M Rhodes, International Escherichia and Klebsiella Centre, Statens Seruminstitut, Artillerivej 5, DK-2300 Copenhagen S, Denmark

Accepted for publication 24 February 1986 no symptoms at all. ${ }^{2}$

Despite the high incidence of trichomoniasis, little is known about the mechanism of the onset of the severe inflammation and cytopathogenic changes that can be characteristic of the disease. ${ }^{1}$ Several reports have been published, however, on the cytopathogenic effect of freshly isolated $T$ vaginalis on various types of mammalian cells or human cancer cells in tissue culture. ${ }^{3-6}$

To investigate the cytotoxic effect of different strains of freshly isolated $T$ vaginalis in vitro, we decided to explore the use of normal human vaginal epithelial cells as target cells. In this report we describe morphological studies on the cytotoxicity of freshly isolated $T$ vaginalis compared with $T$ vaginalis strain 1711 , which was previously used in extensive studies on the ultrastructure of $T$ vaginalis, ${ }^{7}$ towards primary cultures of human vaginal epithelial cells. 


\section{Materials and methods}

\section{STRAINS OF PARASITE}

$T$ vaginalis strain 1711 , originally described by Nielsen, ${ }^{7}$ was obtained from a cryopreserved stock in the Neisseria Department, Statens Seruminstitut. $T$ vaginalis strain 130354 was freshly isolated from the genital tract of a patient with severe symptoms of trichomoniasis, and $T$ vaginalis strain 10 was obtained as a fresh isolate from the Neisseria Department. All strains of $T$ vaginalis were maintained in Diamond's medium $^{8}$ containing agar and were transferred daily into fresh Diamond's medium with agar. At least one week before the experiments the trichomonads were transferred to Diamond's medium without agar. The growth characteristics of $T$ vaginalis strains 1711 and 130354 in this medium were assessed by counting the number of trichomonads in a haemocytometer at different times within 50 hours after inoculation of the medium.

\section{EPITHELIAL CELLS}

Vaginal epithelial tissue was obtained from the vaginal cuff that is removed with the uterus at operation on patients requiring a hysterectomy for non-malignant disease. Immediately after surgery the tissue was placed in Eagle's minimum essential medium (EMEM) supplemented with $10 \%$ fetal calf serum, $0.01 \%$ streptomycin, and $0.002 \%$ neomycin (EMEM+). On arrival at the laboratory, the epithelial tissue was dissected from the connective tissue. The epithelial sheet was cut into cubes $\left(8 \mathrm{~mm}^{3}\right)$, which were placed in chicken embryo extract (Gibco, Europe). Small drops of chicken plasma (Gibco, Europe) were placed on the bottom of each well of a Linbro tissue culture plate ( 24 wells). The epithelial explants were then transferred to the drops of plasma, and the plasma was allowed to clot. When the clot had formed $2 \mathrm{ml}$ EMEM + was added to each well, and the plate was incubated at $37^{\circ} \mathrm{C}$ in an atmosphere of $5 \%$ carbon dioxide. The medium was changed after one week and thereafter twice a week. A confluent sheet of epithelial cells was seen after four weeks.

\section{MORPHOLOGICAL STUDIES OF MONOLAYERS}

AFTER INOCULATION WITH $T$ VAGINALIS

Confluent monolayers were washed twice with EMEM, and $2 \mathrm{ml}$ fresh EMEM+ was added. Trichomonads were harvested by centrifugation at 400 $x g$ for five minutes, resuspended in Diamond's medium without agar, and counted in a haemocytometer.

\section{Light microscopy}

The following concentrations of $T$ vaginalis were used: $10^{4}, 10^{5}, 10^{6}$, and $10^{7}$ per ml Diamond's medium without agar, $100 \mu l$ of these suspensions was added to the epithelial monolayers. All experiments were repeated three times, and triplicate estimations were carried out within each experiment. The Linbro plates were incubated at $37^{\circ} \mathrm{C}$ for 24 hours in an atmosphere of $5 \%$ carbon dioxide. The condition of the cells throughout the incubation period was observed by phase contrast microscopy. After incubation the epithelial monolayers were washed with phosphate buffered saline (PBS), pH 7.0, and fixed and stained with Wright's stain. Cytotoxicity was assessed as follows: $0=$ no damage to the epithelial cells; $1=10$ $25 \%$ destruction; $2=25-50 \%$ destruction; $3=50$ $75 \%$ destruction; and $4=75-100 \%$ destruction.

\section{Electron microscopy}

Monolayers of epithelial cells were inoculated with $100 \mu l$ of $10^{6} / \mathrm{ml} T$ vaginalis organisms $6-24$ hours before they were processed for electron microscopy. Each culture had $0.5 \mathrm{ml}$ Karnovsky's fixative ${ }^{9}$ added, and five minutes later this was replaced by Karnovsky's fixative dilutred 1:2 with distilled water. After one hour the cultures were washed in $0.1 \mathrm{~mol} / 1$ cacodylate buffer, $\mathrm{pH} 7 \cdot 3$, post fixed in $1 \%$ osmium oxide in $0 \cdot 1$ mol/1 cacodylate, $\mathrm{pH} 7 \cdot 3$, for three hours, washed briefly, and dehydrated in graded ethanol followed by $100 \%$ propylene dioxide. After this treatment the epithelial cell monolayer became loose. The cells were transferred to a mixture of equal parts of propylene dioxide and epon. The cells were rolled up using an orange stick and embedded in Beam capsules with the roll standing upright.

In some experiments all fixatives, buffer solutions, and ethanol solutions up to $70 \%$ alcohol contained $0 \cdot 1 \%$ ruthenium red.

Ultrathin sections were post stained with uranyl acetate and lead citrate.

\section{Results}

\section{LIGHT MICROSCOPY}

Growth characteristics

$T$ vaginalis strain 130354 grew more slowly than strain 1711 in Diamond's medium without agar and did not attain the same total number of trichomonads. The peak numbers reached 30 hours after inoculation with $5 \times 10^{5} T$ vaginalis were about $4 \times 10^{6}$ for strain 130354 and $10^{7}$ for strain 1711. Thereafter strain 1711 died rapidly and there were no viable cells 40 hours after inoculation, whereas 60 hours were required before strain 130354 was dead.

\section{Interaction of $T$ vaginalis with monolayers}

$T$ vaginalis 1711 organisms retained their ovoid shape after addition to epithelial cell cultures, and moved about actively, either singly or in clumps of up to several hundreds of trichomonads. Adherence to the 


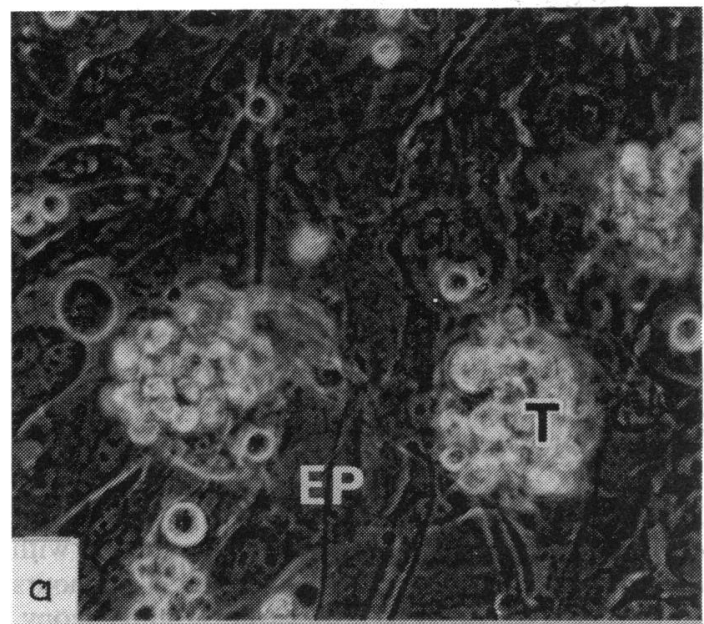

monolayers was rarely seen even after incubation for 24 hours (fig 1(a), (b), and (c)). Only a few $T$ vaginalis 1711 organisms were visible in the fixed stained preparations, most having been washed away.

$T$ vaginalis 130354 and 10 organisms also moved about actively for the first half hour after inoculation, but after one hour these trichomonads settled on the epithelial cells singly and then showed amoeboid movements with projecting pseudopodia. After 48 hours they often grew into sheets partly covering the monolayer (fig 2(a), (b), and (c)). These two strains adhered to the epithelial monolayer, and most of the trichomonads still adhered to the epithelial cells after being washed and fixed.

The adherence of $T$ vaginalis to the monolayers seemed to be associated with their cytotoxic activity. Cell destruction caused by the freshly isolated strains was thus initially confined to areas with adhering trichomonads. The table shows that $T$ vaginalis strain 1711 , which did not adhere to the monolayers, only caused a demonstrable cytotoxic effect towards epithelial cells when $10^{5}$ trichomonads were added per well. In contrast, $T$ vaginalis strain 130354 showed a

TABLE Cytotoxic effect of two different strains of Trichomonas vaginalis on normal human vaginal epithelial cells

\begin{tabular}{|c|c|c|}
\hline \multirow{2}{*}{$\begin{array}{l}\text { No of } T \text { vaginalis } \\
\text { micro-organisms } \\
\text { added per well }\end{array}$} & \multicolumn{2}{|c|}{ Cytotoxic effect * of strains: } \\
\hline & 1711 (laboratory) & 130354 (fresh) \\
\hline $10^{3}$ & 0 & 1 \\
\hline $10^{4}$ & 0 & $3-4$ \\
\hline $10^{5}$ & 1 & 4 \\
\hline $10^{6}$ & 3 & 4 \\
\hline
\end{tabular}

$* 0=$ no damage to the epithelial cells; $1=10-25 \%$ destruction; $2=$ $25-50 \%$ destruction; $3=50-75 \%$ destruction; $4=75-100 \%$ destruction.

Experiments were carried out in triplicate. Figures are averages of two identical experiments carried out in triplicate.

visible cytotoxic effect at a concentration of $10^{3}$ trichomonads per well, and more than $10^{3}$ organisms per well totally destroyed the monolayers. The cytotoxic effect of strain 10 (data not shown) was similar to that of strain 130354 .

FIG 1 Phase contrast light micrographs of confluent monolayer of normal human vaginal epithelial cells (a) immediately, (b) 31/2 hours, and (c) 22 hours after inoculation with $10^{4} \mathrm{~T}$ vaginalis strain 1711. At all times most trichomonads $(T)$ were aggregated and non-adherent and epithelial cells (EP) were undamaged. 
FIG 2 Phase contrast light micrographs of confluent monolayer of normal human vaginal epithelial cells (a) immediately, (b) $3 \frac{12}{2}$ hours, and (c) 22 hours after inoculation with $10^{4} T$ vaginalis strain 130354. Immediately after inoculation some trichomonads adhered to epithelial cells and became amoeboid (A). After three and $a$ half hours most parasites had adhered and were amoeboid (A) but epithelial cells $(E P)$ were undamaged. After 22 hours trichomonads lay in sheets over large lesions $(L)$ in the epithelial monolayer. Epithelial cells (EP), which seemed to be normal, were present in unparasitised areas.

\section{ELECTRON MICROSCOPY}

Electron miscroscopy confirmed the light microscopy finding that strain 1711 , in contrast with strains 130354 and 10 , was not retained on the monolayers of epithelial cells after fixation. $T$ vaginalis strain 1711 was never observed in intimate contact with epithelial cells, which as a rule showed normal ultrastructural morphology and intact cell membranes.

$T$ vaginalis 130354 and 10 organisms acquired an amoeboid shape and were often flattened out in intimate contact with the surface of epithelial cells, with neighbouring $T$ vaginalis, or with the plastic on the bottom of the culture well (fig 3 and 4). They were also observed on, between, or under the epithelial cells (figs 3 and 4).

The gap between the cell membranes of trichomonads and epithelial cells that were in close proximity varied, but was never less than $20 \mathrm{~nm}$. At the site of contact the cytoplasm of the trichomonads consisted almost exclusively of a network of microfilaments (fig 5). Sometimes endocytic vesicles with engulfed material were noted. Ruthenium red stained the surface of $T$ vaginalis irrespective of whether the trichomonad was in intimate contact with other cells or the plastic on the bottom of the culture well or was free in the medium (fig 4).

Vaginal epithelial cells incubated with freshly isolated $T$ vaginalis were affected to different degrees depending on the number of $T$ vaginalis organisms and the period of incubation. Six hours after inoculation many epithelial cells in intimate contact with $T$ vaginalis still had a normal cell membrane and cytoplasmic ultrastructure (fig 4), whereas after 24 hours the outer membrane of the epithelial cells in contact with $T$ vaginalis was broken and the cytoplasm only contained a few organelles (figs 3 and 5).

\section{Discussion}

Several reports have indicated that $T$ vaginalis can be cytotoxic towards cells in tissure cultures. ${ }^{13-6}$ These target cells originated either from different animal species or from human tumours. Our studies using
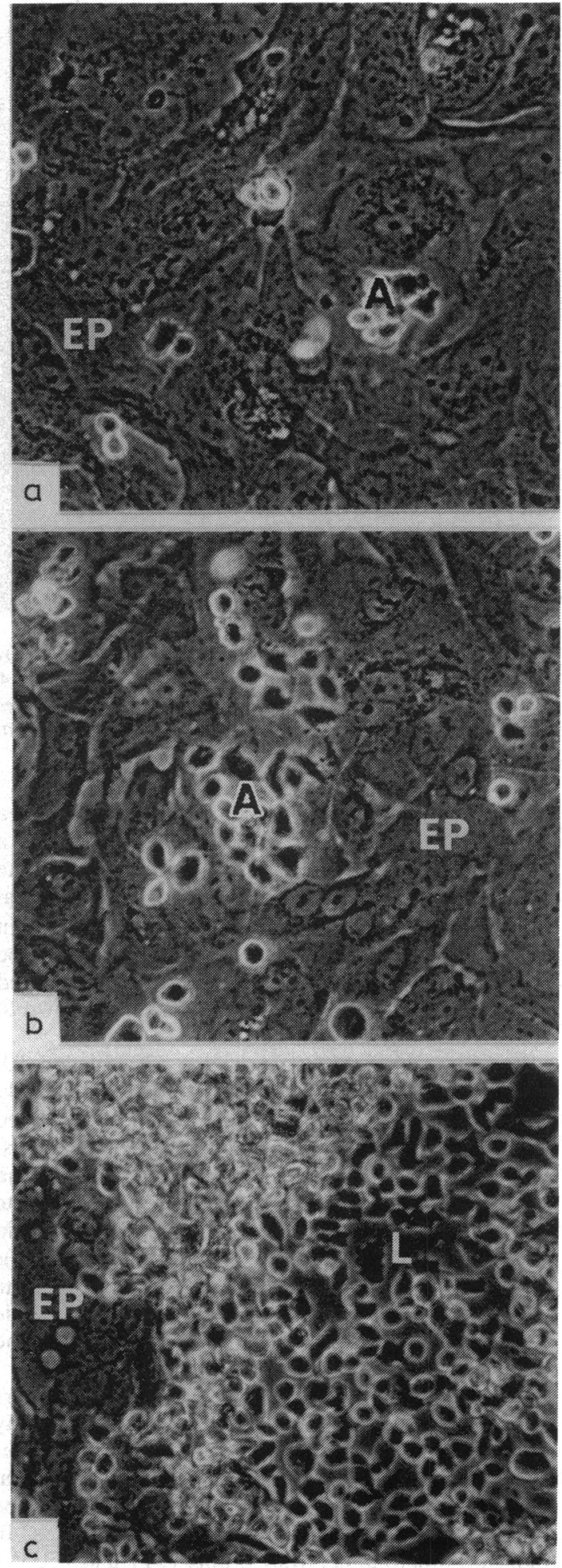


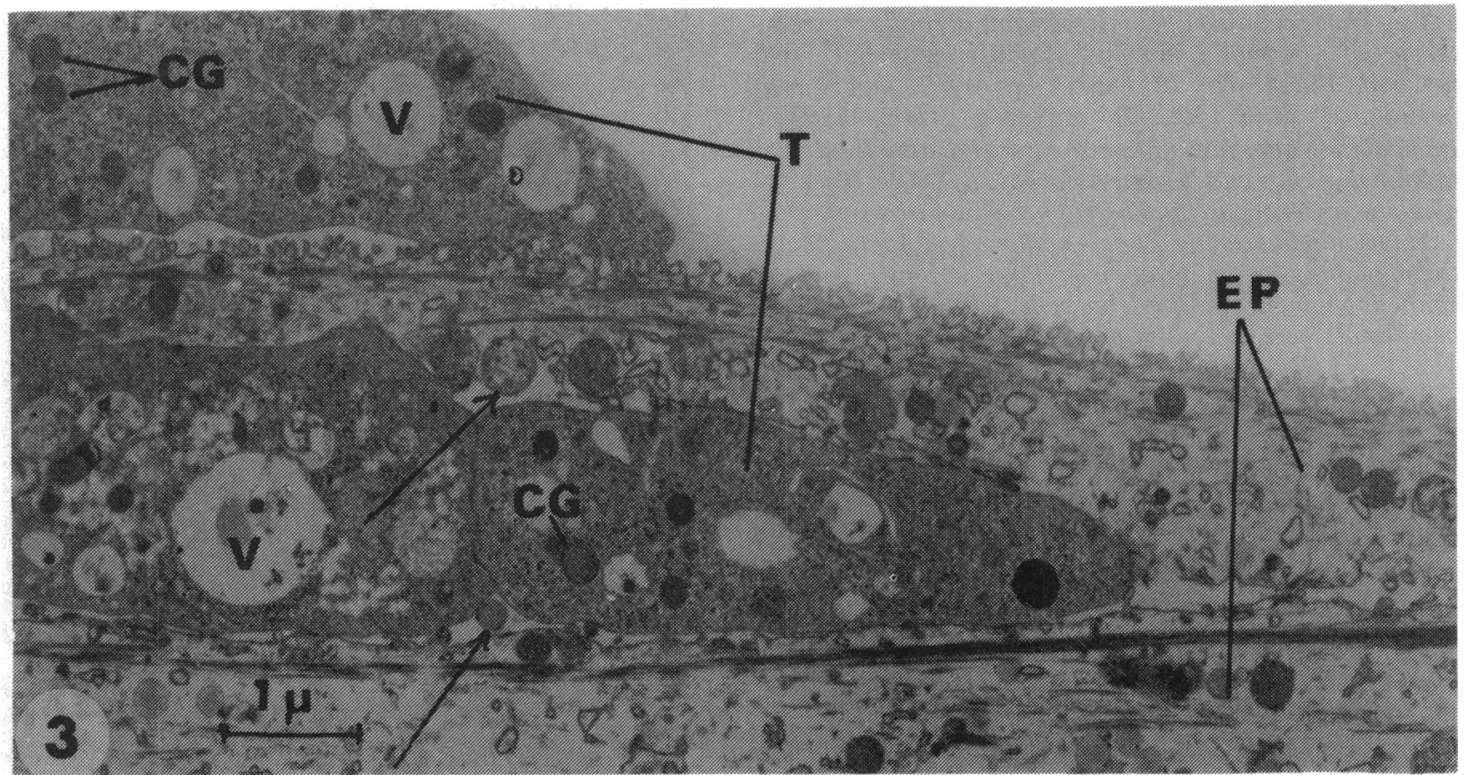

FIG 3 Electron micrograph of vaginal epithelial cell culture 24 hours after inoculation with T vaginalis strain 130354. Note flattened trichomonads (T) on top of and intercalated between epithelial cells (EP). Epithelial cell membrane was

normal human vaginal epithelial cells showed that a strain of $T$ vaginalis (130354) freshly isolated from a patient with severe symptoms and a fresh isolate (strain 10) caused extensive disruption of the epithelial cell monolayer, whereas a strain (1711) maintained in axenic cultures for several years did not. This may be due to the fact that strain 1711 had been passaged frequently in Diamond's medium, which is known to cause a loss in pathogenicity. ${ }^{1}$

The cytopathogenicity of strains 130354 and 10 , which was confined to focal areas, was clearly associated with the fact that the trichomonads settled on the epithelial layer and became attached to the cells. This result agrees with those of other authors, ${ }^{13-6}$ who observed that physical contact between the parasite and target cells was necessary for cell destruction in vitro. It is still not known, however, whether the cytotoxic mechanism is "mechanical" or is due to membrane bound lytic factors. A recent report on the haemolytic activity of trichomonads might support the latter hypothesis. ${ }^{10}$

It has also been suggested that chemical factors released into the culture medium by trichomonads may be responsible for or augment cell destruction. ${ }^{11}$ In our experiments, however, the cell destruction was focal and some normal epithelial cells always seemed to be present after exposure to the pathogenic strains. In addition, supernatants from strains 1711 and 130354 broken and cytoplasmic organelles were present in intracellular space (arrows). CG denotes chromatic granules (hydrogenosomes) and $V$ denotes large food vacuoles in trichomonads.

of trichomonads used in the present studies had no effect on the human vaginal epithelial cells (own unpublished observations). Similar observations have been made by others. ${ }^{36}$ This implies that it is unlikely that release of a toxin into the culture medium is responsible for the destruction of the epithelial cell monolayers. Furthermore, if a toxin is produced by the protozoa, then it is either present at a very low level, acts locally, or is inhibited by the medium.

The electron microscopic observation that freshly isolated $T$ vaginalis organisms with amoeboid morphology attach to monolayers of human vaginal epithelial cells in vitro is similar in most respects to the results of earlier electron microscopic studies on vaginal biopsy specimens from patients with trichomoniasis. ${ }^{12}$ The only difference noted was that in vaginal biopsy specimens trichomonads were exclusively attached to necrotic superficially located cells, whereas in cultures inoculated in vitro with $T$ vaginalis intimate contact between trichomonads and apparently unaltered epithelial cells was observed after six hours. This may imply that the cytotoxicity of $T$ vaginalis towards epithelial cells is a "slow" process requiring several hours of contact with the target cell.

Intimate contact in vitro between $T$ vaginalis and epithelial cells was apparently required for cytotoxicity to occur, but the fine structure of the zone of 


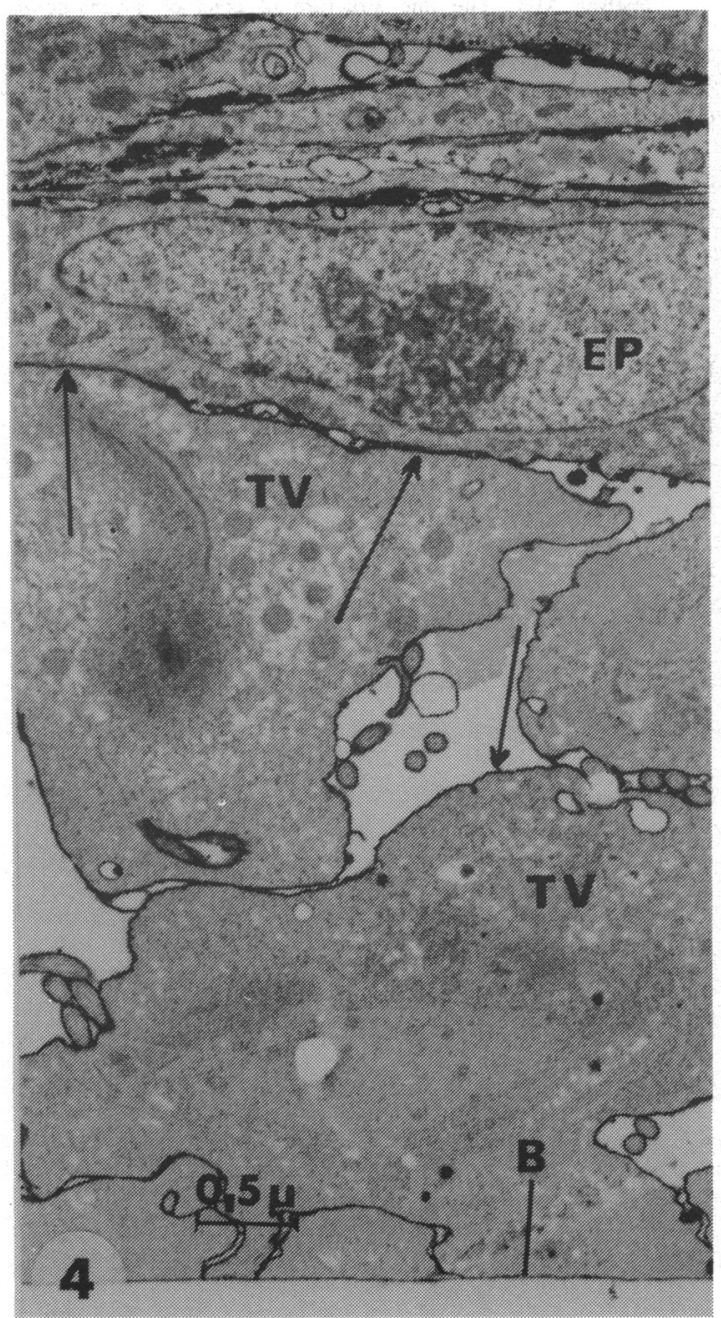

FIG 4 Electron micrograph of cell culture six hours after infection with $T$ vaginalis strain 10 . Trichomonad cells $(T)$ in intimate contact with a detached undamaged epithelial cell $(E P)$ on bottom of well (B). Uniform ruthenium red staining (arrows) of all cell surfaces implied that there was no tight junction contact between closely apposed cell membranes.

contact gave no indication of how this might take place.

Inoculation of monolayers in vitro, as described here, may provide a model system for studying the pathogenicity of $T$ vaginalis. Other local factors prevalent in the vagina, however, such as the influx of granulocytes, the release of enzymes, and the effect of other micro-organisms, must be taken into account to

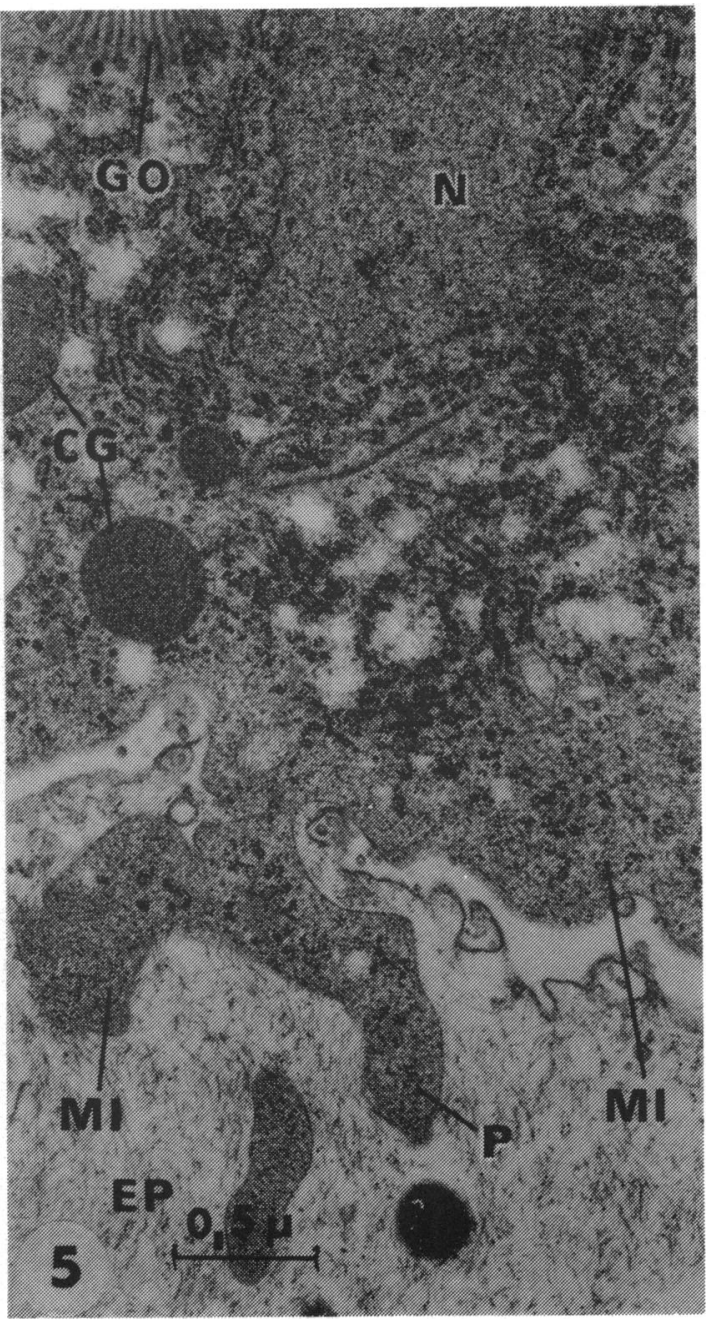

FIG 5 Electron micrograph of cell culture 24 hours after inoculation with $T$ vaginalis strain 130354. $T$ vaginalis pseudopodia $(P)$ project into cytoplasm (EP) of partially disintegrated epithelial cell. $M I$ denotes microfilaments, $N$ nucleus, GO golgi zone, and $C G$ chromatic granules (hydrogenosomes) of $T$ vaginalis.

explain the onset of the severe inflammatory response that is characteristic of the disease.

We are indebted to Dr Lebech, head of the gynaecological department, Frederiksberg Hospital, for the specimens of vaginal tissue, and to Dr Mary Andersen, specialist in gynaecology, for the $T$ vaginalis isolated from a patient with severe trichomoniasis. 


\section{References}

1. Honigberg BM. Trichomonas of importance in human medicine. In: Kreier JP, ed. Parasitic protozoa. Vol 2. New York: Academic Press, 1978:275-454.

2. Krieger JN. Urologic aspects of trichomoniasis. Investigative Urology 1981;18:411-7.

3. Farris VK, Honigberg BM. Behaviour and pathogenicity of Trichomonas vaginalis Donné in chick liver cell cultures. $J$ Parasitol 1970;56:849-82.

4. Alderete JF, Pearlman E. Pathogenic Trichomonas vaginalis cytotoxicity to cell culture monolayers. British Journal of Venereal Diseases 1984;60;99-105.

5. Heath E. Behaviour and pathogenicity of Trichomonas vaginalis in epithelial cell cultures. British Journal of Venereal Diseases 1981;57:106-17.

6. Christian RT, Miller NF, Ludovici PP, Riley GM. A story of Trichomonas vaginalis in human cell culture. Am J Obstet Gynecol 1963;85:947-54.
7. Nielsen MH. The fine structure of cells of Trichomonas vaginalis Donne obtained from the exponential phase of growth and from stationary culture. Acta Pathol Microbiol Scand B 1976;84:205-16.

8. Diamond LS. The establishment of various trichomonads of animal and man in axenic cultures. $J$ Parasitol 1957;43:48898.

9. Karnovsky MJ. A formaldehyde glutaraldehyde fixative of high osmolarity for use in electron microscopy. J Cell Biol 1965;27:137A.

10. Krieger JN, Poisson MA, Rein MV. Beta-hemolytic activity of Trichomonas vaginalis correlates with virulence. Infect Immun 1983;41:1291-5.

11. Hogue MJ. The effect of Trichomonas vaginalis on tissue culture cells. American Journal of Hygiene 1943;37:142-52.

12. Nielsen MH, Nielsen R. Electron microscopy of Trichomonas vaginalis Donné. Interaction with vaginal epithelium in human trichomoniasis. Acta Pathol Microbiol Scand B 1975;83:30520. 